\title{
REHABILITACIÓN CARdIACA EN PACIENTES CON CARDIOPATÍA CORONARIA EN EL INSTITUTO NACIONAL CARDIOVASCULAR DE ESSALUD SETIEMBRE 2017-FEBRERO 2018
}

\author{
CARDIAC REHABILITATION \\ IN PATIENTS WITH \\ CORONARY CARDIOPATHY \\ AT THE CARDIOVASCULAR \\ NATIONAL INSTITUTE OF \\ ESSALUD SEPTEMBER 2017- \\ FEBRUARY 2018 \\ Marco Antonio Heredia Ñahui ${ }^{1}$ \\ Rosalia Fernández C. ${ }^{2}$ \\ Julia Fernández C. $^{3}$ \\ Mariana Heredia $\tilde{N}^{4}$ \\ Ximena Gordillo M. ${ }^{5}$ \\ Rocio Palomino ${ }^{\circ}$ \\ Renzo Soca ${ }^{7}$
}

1. Especialista en Cardiología. Instituto Nacional Cardiovascular - INCOR - Essalud.

2. Magister en Gerencia de Servicios de salud. Coordinadora UFRC-INCOR-Essalud Jefe de la Oficina de Docencia e Investigación INCOR-Essalud Profesor Asociado UNMSM.

3. Especialista en psicología clinica y de la salud. Psicóloga Unidad Funcional de Rehabilitación Cardiaca.

4. Médico Cirujano.

5. 6.7. Especialista en Cardiología. Instituto Nacional Cardiovascular - INCOR - Essalud.

\section{RESUMEN}

Las enfermedades cardiovasculares (ECV), constituyen un problema de salud pública a nivel mundial; en EsSalud, se ubica en séptimo puesto de las patologías con mayor carga de enfermedad. Los programas de rehabilitación cardiaca basados en ejercicios equipan un conjunto de intervenciones diseñadas para mejorar la recuperación del paciente con problemas cardiovasculares agudos o crónicos, los cuales mejoran la capacidad funcional, calidad de vida y ayudan al control de los factores de riesgo. OBJETIVO: Evaluar el efecto de un Programa de Rehabilitación Cardiaca (PRC) en pacientes con enfermedad coronaria. MATERIALES Y MÉTODOS: EI estudio fue de tipo observacional, analítico, retrospectivo y longitudinal. La población estuvo constituida por pacientes que ingresaron al Programa de Rehabilitación Cardiaca del INCOR-ESSALUD entre los meses de setiembre a diciembre del 2017 con diagnóstico de enfermedad coronaria y que completaron las evaluaciones pertinentes. RESULTADOS: Se analizaron 109 historias clínicas de pacientes con enfermedad coronaria la mayoría de sexo masculino y con hipertensión arterial como el principal factor de riesgo cardiovascular. Se encontró que luego de completar la PRC, los participantes disminuyeron la circunferencia abdominal $(p=0.000)$, la presión arterial $(p=0.000)$ y mejoraron su consumo de oxígeno estimado en METS ( $p=0.000)$; asimismo hubo una reducción de la obesidad abdominal $(p=0.008)$ y mejoría de la clase funcional ( $p=0.000$ ); además existe una mejoría significativa en la calidad de vida de los pacientes $(p=0.000)$. CONCLUSIONES: EI PRC tiene efectos significativos sobre la obesidad abdominal, mejoría del consumo de oxígeno estimado, capacidad funcional y calidad de vida de los pacientes con enfermedad coronaria.

Palabras Claves: Calidad de vida, capacidad funcional, enfermedad coronaria, rehabilitación cardiaca.

\section{ABSTRACT}

Cardiovascular diseases (CVD) are a public health problem worldwide; in EsSalud, it ranks seventh in the pathologies with the highest burden of disease. Cardiac rehabilitation programs based on exercises equip a set of interventions designed to improve the recovery of patients with acute or chronic cardiovascular problems, which improve functional capacity, quality of life and help control risk factors. OBJECTIVE: To evaluate the effect of a Cardiac Rehabilitation Program (CRP) in patients with coronary heart disease. MATERIALS AND METHODS: The study was observational, analytical, retrospective and longitudinal. The population consisted of patients who entered the Cardiac Rehabilitation Program of INCOR-ESSALUD between the months of September to December 2017 with a diagnosis of coronary disease and who completed the pertinent evaluations. RESULTS: A total of 109 clinical histories of patients with coronary disease were analyzed, most of them were male and hypertension was the main cardiovascular risk factor. It was found that after completing the PRC, the participants decreased the abdominal circumference $(p=0.000)$, blood pressure $(p=0.000)$, as well as their oxygen consumption estimated in METS $(p=0.000)$. Also, there was a reduction in abdominal obesity $(p=0.008)$ and improvement in functional class $(p=0.000)$. Furthermore, there is also a significant improvement in the quality of life of patients $(p=0.000)$. CONCLUSIONS: PRC has significant effects on abdominal obesity, improvement of estimated oxygen consumption, functional capacity and quality of life of patients with coronary heart disease.

Keywords: Quality of life, functional capacity, coronary disease, cardiac rehabilitation. 


\section{INTRODUCCIÓN}

Las enfermedades cardiovasculares (ECV), constituyen un problema de salud pública a nivel mundial, responsable de más de 17.3 millones de muertes al año (1). En los Estados Unidos se calcula que 27.6 millones de norteamericanos (11.5\%) está diagnosticado con enfermedad cardiovascular y el $43.8 \%$ de las muertes por estas enfermedades son causadas por la enfermedad coronaria ${ }^{(2)}$. En el Perú representan más de 5455 muertes prematuras anuales, siendo la primera causa de éstas, la enfermedad isquémica del corazón con el $31 \%{ }^{(3)}$. En EsSalud, se ubica en séptimo puesto de las patologías con mayor carga de enfermedad y con afectación a los mayores de 60 años ${ }^{(4)}$.

Los programas de rehabilitación cardiaca basados en ejercicios (PRCBE) equipan un conjunto de intervenciones diseñadas para mejorar la recuperación del paciente con eventos cardiovasculares agudos 0 pacientes con enfermedad cardiovascular estable ${ }^{(5)}$. Los Programas de Rehabilitación Cardiaca en su fase ambulatoria o Fase II duran en promedio entre 4 a 12 semanas y se caracterizan por ejercicios aeróbicos, de resistencia, flexibilidad y equilibrio de 8 semanas de duración ${ }^{(6)}$.

La inclusión frecuente de los programas de entretenimiento, tanto aeróbico como de resistencia, brindan mayor autonomía en las actividades de la vida diaria y, asimismo, mejoran la percepción de mejoría y capacidad funcional ${ }^{(7)}$. Adicionalmente, la actividad física tiene efectos psicológicos que mejoran la interacción social, disminuyen el estrés emocional y depresión ${ }^{(1)}$; por lo tanto, mejoran la calidad de vida de los pacientes, tanto en los que son intervenidos de manera invasiva como no invasiva ${ }^{(1)}$. Este puede ser medido a través de instrumentos como el QLMI-2, el cual es un instrumento específico para medir la calidad de vida a pacientes con diversas presentaciones y síntomas de enfermedades cardiovasculares ${ }^{(7)}$.

En el Perú no existen estudios sobre el beneficio de los programas de rehabilitación cardiaca en enfermedades cardiovasculares y mucho menos en pacientes con cardiopatía coronaria crónica. Además, son pocos los establecimientos de salud en donde se han implementado como prevención secundaria y terciaria en estas patologías, en base a los beneficios hallados en estudios internacionales.

El objetivo del estudio fue evaluar el efecto de un Programa de Rehabilitación Cardiaca en pacientes con enfermedad coronaria.

\section{MATERIALES Y MÉTODOS}

El estudio fue de tipo observacional, analítico, retrospectivo y longitudinal. Se revisaron las historias clínicas de los pacientes que realizaron rehabilitación cardiaca en el Instituto Nacional Cardiovascular Carlos Alberto Peschiera Carrillo durante los meses de setiembre 2017 y diciembre 2017, y se incluyeron a todos los pacientes con diagnóstico de enfermedad coronaria aguda y cardiopatía coronaria crónica, mayores de 18 años. Se excluyeron pacientes que no completaron el programa, que fueron dados de alta antes de las 8 semanas o que no tuviesen todas las evaluaciones completas.

Los pacientes fueron evaluados siguiendo el protocolo institucional para la Fase II de Rehabilitación Cardiaca. Se les realizó una primera consulta cardiológica para conocer la historia clínica del paciente y detectar sus factores de riesgo cardiovascular. Realizaron los exámenes auxiliares de ingreso según protocolo, laboratorio y prueba funcional tipo prueba de esfuerzo graduada. En la segunda consulta, se evaluó los resultados de las pruebas y se decidió su ingreso al programa. Asimismo, recibieron intervención por el equipo multidisciplinario: médico cardiólogo, enfermera, psicóloga y nutricionista para afianzar la educación y adherencia del paciente y familiares al programa. Se les entregó un cronograma de actividades para las 8 semanas de permanencia, que incluía una charla educativa, dos talleres de nutrición, dos talleres de psicología y dos terapias de campo. Durante la primera sesión de rehabilitación cardiaca, "sesión 0", se les aplicó el test de calidad de vida QLMI2; también se hicieron las medidas antropométricas iniciales que incluyeron el peso, talla, índice de masa corporal (IMC) y circunferencia abdominal (CA). Según estas medidas se definió como bajo peso, eutrófico, sobrepeso y obesidad. Se consideró también la obesidad abdominal según las recomendaciones de la Federación Americana de Diabetes ${ }^{(8)}$. Luego de transcurridas las 08 semanas en el programa, se les realizó una segunda evaluación mediante laboratorio, prueba funcional tipo prueba de esfuerzo graduada y se les aplicó nuevamente el cuestionario QLMI-2. Se realizó el procesamiento y análisis de los datos con Microsoft Excel versión 2013 y IBM SPSS Statistics versión 20.0. Finalmente, se empleó estadística descriptiva para evaluar las características poblacionales y las pruebas de $T$ de Student y chi cuadrado para comparar la evaluación inicial y final. 


\section{RESULTADOS}

Se revisaron las historias de los pacientes que ingresaron al Programa de Rehabilitación Cardiaca del Instituto Nacional Cardiovascular - INCOR, durante los meses de setiembre a diciembre del 2017, encontrándose 188 historias de pacientes con diagnóstico compatible con enfermedad coronaria, de las cuales 100 historias fueron de enfermedad coronaria crónica y 88 de enfermedad coronaria aguda. De los 100 pacientes con enfermedad coronaria crónica fueron excluidos 6 historias por diversos motivos, de los 94 pacientes que completaron el Programa se excluyeron 37 historias, quedando al final 57 historias elegibles. Por otro lado, de las 88 historias de pacientes con enfermedad coronaria aguda, fueron excluidos inicialmente 11 historias por diversos motivos, quedando 77 historias de pacientes que completaron el programa, de las cuales se excluyeron 15 historias, quedando 62 casos para analizar.

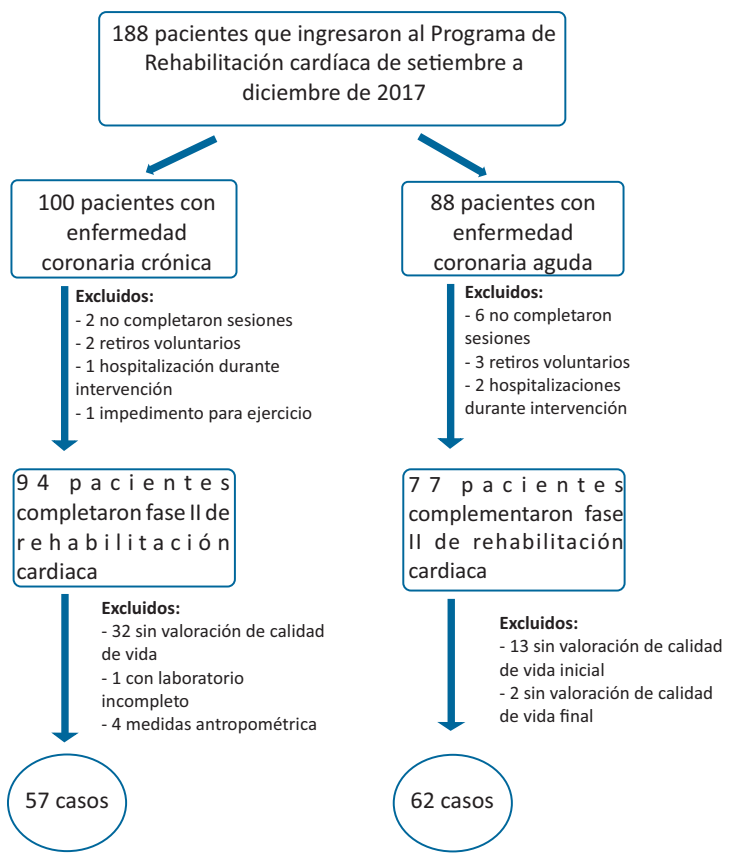

Figura 1. Fluxograma de selección de historias clínicas analizadas.

Se analizaron 62 (52.1\%) historias de pacientes con diagnóstico de enfermedad coronaria aguda y 57 historias de pacientes con diagnóstico de enfermedad coronaria crónica (47.9\%); 106 pacientes fueron varones (89.1\%) y 13 mujeres (10.9\%). Los factores de riesgo relacionados a estos pacientes fueron hipertensión arterial en 72 pacientes (60.5\%), sobrepeso 61 pacientes (51.3\%), dislipidemia en 60 pacientes $(50.4 \%)$, diabetes mellitus en 31 pacientes (26.1\%), entre otros factores de riesgo. En la Tabla 1 presentamos el resumen de las características principales de nuestra población.

Tabla 1. Características generales de la población

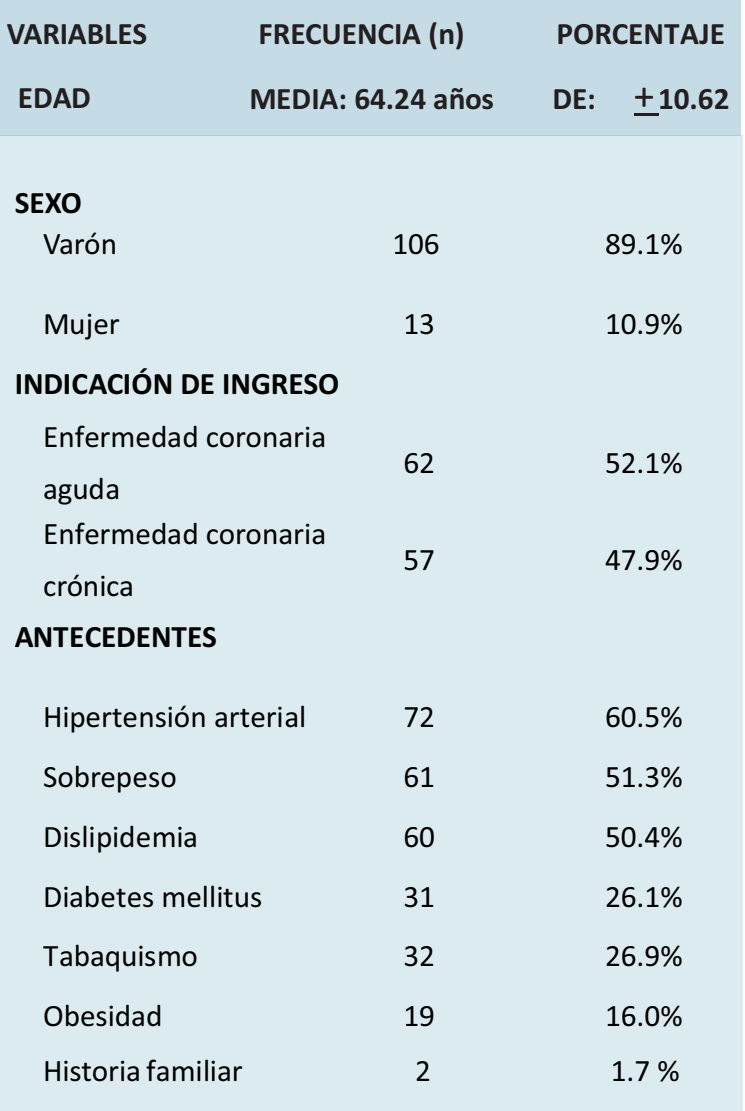

En la Tabla 2 mostramos las variables antropométricas medidas antes y después de la Rehabilitación Cardiaca, podemos observar que no existe una variación significativa entre el Índice de Masa Corporal (IMC) de ingreso y de alta $(P=0.180)$, pero sí existe una variación significativa entre la Circunferencia Abdominal (CA) de ingreso y al alta $(P=0.000)$. Asimismo, mostramos la diferencia entre la presión sistólica y diastólica comparándolas antes y después del programa, mostrando una reducción significativa de ambas luego de completar el Programa de Rehabilitación Cardiaca $(\mathrm{P}=0.000$ en ambos casos). También, existe una mejoría de la capacidad funcional estimada a través de la estimación del consumo de oxigeno expresados en METS $(P=0.000)$. Finalmente, mostramos las variables 
bioquímicas, que no presentaron variación estadísticamente significativa.

\begin{tabular}{|c|c|c|c|c|}
\hline \multicolumn{5}{|c|}{ Programa de rehabilitación cardiaca } \\
\hline VARIABLE & DESP & ES DIF & ENCIA & $\underset{P}{\text { VALOR DE }}$ \\
\hline \multicolumn{5}{|l|}{$\begin{array}{c}\text { MEDIDAS } \\
\text { ANTROPOMÉTRICAS }\end{array}$} \\
\hline IMC & 26.45 & 26.21 & -0.24 & 0.180 \\
\hline CA & 96.30 & 94.38 & -1.92 & 0.000 \\
\hline \multicolumn{5}{|l|}{ PRESIÓN ARTERIAL } \\
\hline Sistólica & 126.88 & 119.31 & -7.57 & 0.000 \\
\hline Diastólica & 74.37 & 67.96 & 6.41 & 0.000 \\
\hline \multicolumn{5}{|l|}{$\begin{array}{l}\text { CAPACIDAD } \\
\text { FUNCIONAL }\end{array}$} \\
\hline METS & 6.44 & 7.71 & +1.27 & 0.000 \\
\hline \multicolumn{5}{|l|}{$\begin{array}{l}\text { EXÁMENES DE } \\
\text { LABORATORIO }\end{array}$} \\
\hline Glucosa & 109.09 & 106.72 & -2.37 & 0.250 \\
\hline Ac. Úrico & 5.80 & 5.76 & -0.04 & 0.694 \\
\hline Colesterol & 128.05 & 128.67 & +0.62 & 0.808 \\
\hline HDL & 40.52 & 41.18 & -0.66 & 0.392 \\
\hline LDL & 59.43 & 63.66 & +4.22 & 0.255 \\
\hline TG & 141.01 & 133.56 & -7.45 & 0.151 \\
\hline $\begin{array}{c}\text { Colesterol no } \\
\text { HDL }\end{array}$ & 87.52 & 87.48 & -0.04 & 0.986 \\
\hline
\end{tabular}

En la Tabla 3 se presentan las variables nutricionales; como podemos observar, hubo una mejoría en todas las variables observadas: se redujo los pacientes con bajo peso, aumentaron pacientes con peso normal o eutrófico, se redujeron la cantidad de pacientes con sobrepeso y obesidad. Sin embargo, estos resultados no tuvieron significancia estadística ( $p=0.117)$. Al evaluar la obesidad abdominal por la CA se observó que sí hubo una reducción significativa $(p=0.008)$ de la misma. También, hubo una mejoría de la capacidad funcional estimada en todos los grupos evaluados $(p=$ 0.000).

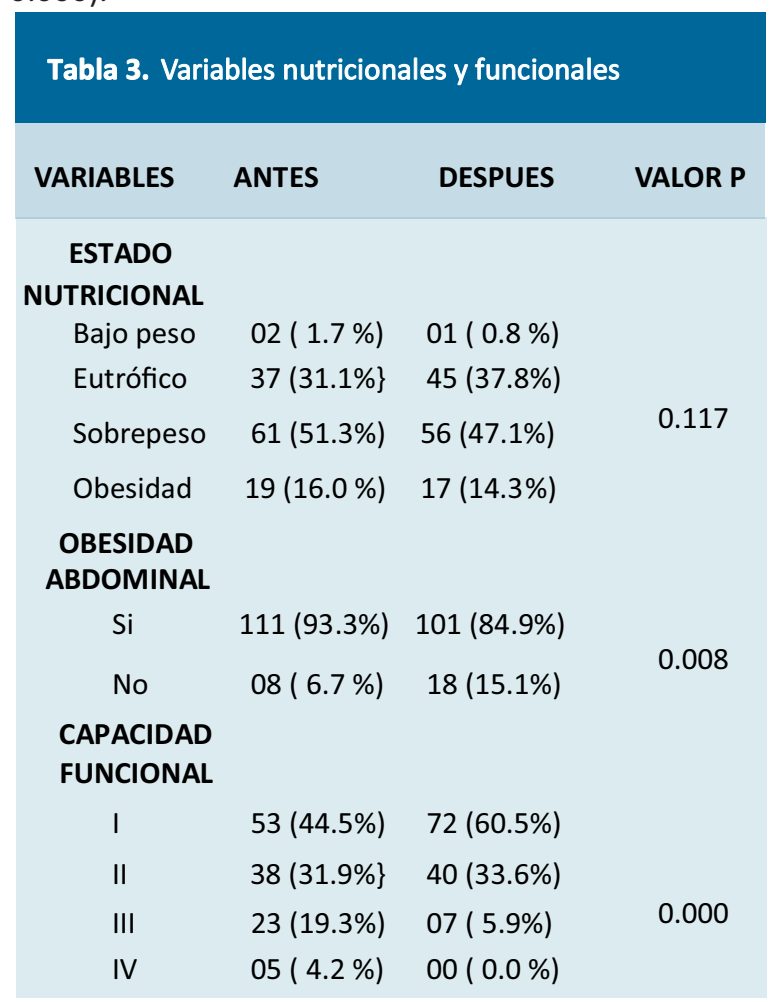

La Tabla 4 muestra el impacto en la Calidad de vida de los pacientes en las dimensiones medidas por el QLMI2. Se muestra una variación significativa de la percepción en la calidad de vida en los pacientes con significancia estadística $(P<0.001)$.

\section{Tabla 4. Impacto en la calidad de vida}

$\begin{array}{ccccc}\text { VARIABLE } & \text { ANTES } & \text { DESPUES } & \begin{array}{c}\text { DIFERENCIAS VALOR } \\ \text { DE MEDIAS }\end{array} & \begin{array}{c}\text { P } \\ \text { DIMENSIÓN }\end{array} \\ \begin{array}{c}\text { EMOCIONAL } \\ \text { DIMENSIÓN } \\ \text { FÍSICA }\end{array} & 5.1 \pm 0.8 & 5.1 \pm 0.6 & +0.3 \pm 0.9 & 0.001 \\ \begin{array}{c}\text { DIMENSIÓN } \\ \text { SOCIAL }\end{array} & 5.6 \pm 1.2 & 5.9 \pm 0.8+0.4 \pm 1.0 & 0.000\end{array}$




\section{DISCUSIÓN}

Nuestro estudio muestra que la mayor parte de pacientes tratados por enfermedad coronaria son varones, esto no difiere con otros estudios comparados donde la población masculina siempre es predominante; además, los pacientes en su mayoría usuarios de programas de rehabilitación cardiaca son de la tercera edad y la mayoría hipertensos ${ }^{(9)(10)}$. Al ser nuestro centro un Instituto especializado y de referencia, la mayoría de nuestros pacientes tuvieron el diagnóstico de ingreso de enfermedad coronaria aguda en relación a los pacientes que portaban enfermedad coronaria crónica.

La rehabilitación cardiaca ha mostrado tener beneficios a nivel cardiovascular, a partir un programa que combina la educación, los cambios de estilo de vida y el seguimiento médico. Esto ha permitido un óptimo control de los factores de riesgo cardiovascular, mejora de la clase funcional y disminución de la morbilidad y mortalidad ${ }^{(1)}$. En este estudio encontramos que el programa de rehabilitación cardiaca logró mejorar la capacidad funcional, controlar la presión arterial y mejorar la calidad de vida de los pacientes.

La reducción significativa de la presión arterial sistólica y diastólica evidencia la importancia del entrenamiento físico sobre la mejora de la función endotelial ${ }^{(11)}$, hallazgos similares a los reportados en otros estudios ${ }^{(9)(10)(11)}$.

La mejoría de la capacidad funcional estimada por METs fue significativa y esto se correlaciona con datos encontrados en otros estudios ${ }^{(9)}(10)$, lo que muestra el impacto del ejercicio sobre la performance cardiovascular del paciente ${ }^{(1)(12)}$.

No hubo reducción significativa de los pacientes que tuvieron sobrepeso y obesidad en nuestro grupo de trabajo. Por otro lado, sí hubo una reducción significativa en los que tuvieron obesidad abdominal según los criterios de la Federación Americana de Diabetes para América Latina ${ }^{(8)}$, lo cual se puede correlacionar a la reducción de la grasa corporal y grasa visceral en nuestro grupo de estudio ${ }^{(13)}$.

No se encontró una reducción significativa en las cifras de colesterol total, HDL-c, LDL-c y triglicéridos medidas en los pacientes. Este resultado difiere con los resultados reportados en el metanálisis de Jolliffe ${ }^{(14)}$, donde se evidenció una reducción significativa en las cifras de colesterol total y LDL-c, mas no un incremento significativo en el HDL-c. Es probable que este hallazgo en nuestro estudio esté relacionado al hecho de que los pacientes ingresan al programa con medicación óptima para el tratamiento de dislipidemia y cifras cercanas o dentro de los rangos esperados.

La capacidad funcional mejoró debido a la mejoría del consumo de oxigeno estimado a través de las pruebas de esfuerzo. Popovic D. y cols. ${ }^{(9)}$ muestran un incremento del consumo de oxígeno, y mejoría de otras variables ergoespirometricas, si bien es cierto no se realizó una medicación cuantitativa del consumo de oxígeno, estos hallazgos son similares.

Nuestro estudio también evidencia un impacto significativo en la calidad de vida de los pacientes en las esferas emocional, física y social, medidas a través del Test de Calidad de Vida QLMI-2. La evaluación de la calidad de vida es un pilar fundamental de los programas de rehabilitación cardiaca y uno de los objetivos de estos programas es contribuir a mejorar la calidad de vida de los pacientes con enfermedades cardiovasculares ${ }^{(3)}$. Yazdani-Bakhsh R y cols. ${ }^{(15)}$ muestran un impacto significativo en la calidad de vida de los pacientes con enfermedad coronaria crónica que van a revascularización percutánea y cirugía, aunque ellos indican que a los 6 meses de procedimiento existe diferencia entre los grupos; no obstante, a los 24 meses se iguala esta diferencia, y en general sí existe una mejoría significativa en la calidad de vida.

\section{CONCLUSIONES}

La Fase II del programa de rehabilitación cardiaca tiene efectos significativos sobre la obesidad abdominal, en la mejoría de la capacidad funcional y ejercicio. Asimismo, contribuye a mejorar el control de la presión arterial en los pacientes con enfermedad coronaria. La Fase II del programa de rehabilitación cardiaca mejora la calidad de vida los pacientes con enfermedad coronaria en su dimensión emocional, física y social.

\section{LIMITACIONES}

Falta de medición directa de grasa corporal total y ergoespirometría cuantitativa.

\section{AGREDECIMIENTOS}

Equipo de Rehabilitación Cardiaca de la Unidad de Rehabilitación Cardiaca del Instituto Nacional Cardiovascular INCOR. 


\section{REFERENCIAS BIBLIOGRÁFICAS}

1. Kachur S, Chongthammakun V, Lavie CJ, De Schutter A, Arena R, Milani RV, Franklin BA. Impact of cardiac rehabilitation and exercise training programs in coronary heart disease. Prog Cardiovasc Dis. 2017 Jun - Jul;60(1):103114. doi:10.1016/j.pcad.2017.07.002. Epub 2017 Jul 6.

2. Benjamin, E. J., Virani, S. S., Callaway, C. W., Chamberlain, A. M., Chang, A. R., Cheng, S., ... \& de Ferranti, S. D. (2018). Heart disease and stroke statistics-2018 update: a report from the American Heart Association. Circulation, 137(12), e67-e492.

3. Organización Panamericana de Salud: Enfermedades cardiovasculares: Perfiles de País^[Internet], n.d. Perú: Perfil de Enfermedades Cardiovasculares. Disponible en: https://www.paho.org/hq/index.php?option=com_topics\& view $=$ rdmore $\&$ cid $=7283 \&$ ltemid $=40876 \&$ lang $=e s$

4. IETSI. Guía de Práctica Clínica de Rehabilitación Cardiaca. $\mathrm{GPCN}^{\circ} \mid 1$, Versión extensa y anexos. Perú, marzo 2018.

5. Braun TL, Wenger KN, Rosenson RS. Cardiac rehabilitation programs. [Monografía en línea]. Waltham, MA: UpToDate Inc. http://www.uptodate.com (Accedido el 21 de Mayo 2018)

6. López-Jiménez, Francisco, et al. Consenso de rehabilitación cardiovascular y prevención secundaria de las sociedades interamericana y sudamericana de cardiología. Revista Uruguaya de Cardiología, 2013, vol. 28, no 2, p. 189-224.

7. Cano CR, Alguacil DI, Alonso MJ, Molero SA, Miangolarra PJ. Programas de rehabilitación cardiaca y calidad de vida relacionada con la salud. Situación actual. Rev Esp Cardiol. $2012 ; 65: 72-9$ - Vol. $65 \mathrm{Nú}$ m.01 D O I: 10.1016/j.recesp.2011.07.016.

8. Aschner P. Obesity in Latin America. Metab. Syndr., Cham:
Springer International Publishing; 2016, p. 33-9. doi: 10.1007/978-3-319-11251-0_4.

9. Popovic D, Kumar N, Chaudhry S, Bagai A, Arena R. Improvements in Key Cardiopulmonary Exercise Testing Variables Following Cardiac Rehabilitation in Patients With Coronary Artery Disease. Journal of Cardiopulmonary Rehabilitation and Prevention 2018;00:1-4.

10. Rosello A.M., Guzmán P.S., Bolaños A.M. Efecto de un programa de rehabilitación cardíaca en la alimentación, peso corporal, perfil lipídico y ejercicio físico de pacientes con enfermedad coronaria. Rev. costarric. cardiol vol.3 n.2 San José Aug. 2001

11. Shen W, Punyanitya M, Chen J, Gallagher D, Albu J, PiSunyer $X$, et al. Waist circumference correlates with metabolic syndrome indicators better than percentage fat. Obesity. 2006;14:727-36.

12. Lawler $P$, Filion K, Eisenberg M. Efficacy of exercise-based cardiac rehabilitation post-myocardial infarction: A systematic review and meta-analysis of randomized controlled trials. Am Heart J 2011;162:571-584.

13. Yazdani-Bakhsh, R., Javanbakht, M., Sadeghi, M., Mashayekhi, A., Ghaderi, H., \& Rabiei, K. (2016). Comparison of health-related quality of life after percutaneous coronary intervention and coronary artery bypass surgery. ARYA atherosclerosis, 12(3), 124.

14. Jolliffe, J. A., Rees, K., Taylor, R. S., Thompson, D., Oldridge, N., \& Ebrahim, S. (2001). Exercise-based rehabilitation for coronary heart disease. Cochrane Database Syst Rev, 1(1).

15. Green D, Hopman M, Padilla J, Laughlin M, Thijssen D. Vascular Adaptation to Exercise in Humans: Role of Hemodynamic Stimuli. Physiol Rev. 2017 Apr; 97(2): 495-528.

\section{Correspondencia}

Marco Antonio Heredia Ñahui

E-mail: marcoheredia_md@hotmail.com

Fecha de recepción: 02 de octubre de 2018

Fecha de aceptación: 15 de febrero de 2019 